\title{
POLARIZATION LIDAR FOR DETECTING DUST ORIENTATION
}

\author{
Alexandra Tsekeri ${ }^{1 *}$, Volker Freudenthaler ${ }^{2}$, George Doxastakis ${ }^{3}$, Josef Gasteiger ${ }^{4}$, Alexandros \\ Louridas $^{3}$, George Georgoussis ${ }^{3}$, Ioannis Binietoglou ${ }^{5}$, Thanasis Georgiou ${ }^{1}$, Zbigniew Ulanowski ${ }^{6}$, \\ and Vassilis Amiridis ${ }^{1}$
}

\author{
${ }^{1}$ IAASARS, National Observatory of Athens, Athens, Greece \\ ${ }^{2}$ Fakultät für Physik, Meteorologisches Institut, Ludwig-Maximilians-Universität, Munich, Germany \\ ${ }^{3}$ Raymetrics S.A., Greece \\ ${ }^{4}$ Faculty of Physics, University of Vienna, Vienna, Austria \\ ${ }^{5}$ National Institute of R\&D for Optoelectronics, Romania \\ ${ }^{6}$ School of Physics Astronomy and Mathematics, University of Hertfordshire, Hatfield, UK \\ *Email: atsekeri@noa.gr
}

\begin{abstract}
We present the preliminary design of a polarization lidar system for detecting oriented dust particles. The system emits linearly- and circularly-polarized light from two lasers and detects the backscattered light, using two telescopes. The emitted light by each laser is detected from both telescopes alternatively, providing in this way two times the signals recorded by a conventional system. The system has also the capability of performing measurements at different zenith angles. Its detection strategy is based on scattering calculations for mixtures of oriented and randomly-oriented dust particles in different atmospheric scenarios.
\end{abstract}

\section{INTRODUCTION}

Dust orientation is an ongoing investigation in recent years [1]. Its potential proof will be a paradigm shift for dust remote sensing, invalidating the currently used simplifications of randomly-oriented particles. Currently, the only signature of particle orientation comes from astronomical polarimetry measurements of dichroic extinction [1], however these measurements provide only an indication of the alignment and refer to column-integrated values, not being capable for vertically-resolved retrievals. Vertically-resolved measurements of dust orientation can be acquired with a polarization sensitive lidar designed to target the off-diagonal elements of the backscattering matrix which are non-zero only when the particles are oriented [2].

Previous studies have demonstrated the feasibility of using circular or linear depolarization lidar measurements to detect the orientation of ice crystals in clouds [3,4,5] and it has been theoretically shown that these techniques can be extended for oriented dust particles [2]. Geier and Arienti [2] showed that although the linear depolarization ratio is sufficient for discerning ice crystal orientation, this is not the case for smaller particles as dust. What they suggested is to use the linear or circular polarized light and detect the backscatter in different polarization planes. With this approach they were able to retrieve the offdiagonal elements of the backscatter phase matrix, which provide information on the asphericity and the orientation of the particles.

We will construct a polarization lidar that will emit linear- and circular-polarized light and detect the linearly- and circularly-polarized components of the backscattered light. The operating wavelength will be at 532 or $1064 \mathrm{~nm}$ for better probing of the dust coarse mode, and the design will follow the high quality EARLINET standards [6]. It will also employ the capability to measure at more than one zenith angle so as to provide more information on the dust orientation and microphysical properties, depending on the angle of the particle orientation.

To achieve highly accurate polarization measurements with high signal to noise ratio 
(SNR), the proposed lidar system will use high power lasers, large aperture telescopes and small receiver fields of view. In order to achieve good SNR in short measurement times the system will be equipped with two laser sources emitting interleaved linear and circular polarized light and two telescopes for their detection. The linearlyand circularly-polarized components of the backscattered light will be measured utilizing a Polarizing Beam Splitter cube (PBS) in one telescope and a circular analyzer (i.e. a QuarterWave Plate (QWP) at $45^{\circ}$ with a PBS) in the other.

The capability of the system in deriving the percentage of oriented dust particles in the atmosphere is investigated herein with a simulated dataset, considering different atmospheric scenarios. The corresponding backscatter matrices are calculated with the Amsterdam Discrete Dipole Approximation (ADDA) [7] for irregularshaped oriented dust particles [8].

\section{METHODOLOGY}

\subsection{System design}

The system design is shown in Fig. 1. The two lasers (L1 and L2) shoot alternatively and their backscattered signals are collected by both Telescopes (T1 and T2). This "2-Lasers/2Telescopes" setup allows the recording of eight signals from the four detectors of the system (D1,
D2, D3 and D4 in Fig. 1). This cost-effective approach provides higher SNR (up to 4 times) and smaller measurement errors due to no moving optics in receiver and transmitter.

L1 emits $45^{\circ}$ linearly-polarized light with respect to the plane of the PBS1. L2 emits linearlypolarized light which is transformed with $\mathrm{QWP}_{\mathrm{L} 2}$ with fast-axis angle of $45^{\circ}$ to circularly-polarized light. The backscattered light collected by T1 is analyzed to its parallel and cross components of its linearly polarized component, recorded by D1 and D2, respectively. The circularly-polarized component of the backscattered light collected by $\mathrm{T} 2$ is transformed to linearly-polarized component, passing through the QWP2 with fastaxis angle of $45^{\circ}$. Further on, this linearlypolarized component is analyzed to the parallel and cross components through the PBS2 and measured at D3 and D4, respectively.

In our analysis we use the ratios of the recorded signals. Specifically, we use the natural logarithms of these ratios, so as to avoid large values in case of denominator values close to zero. The backscattered signals from L1, recorded at D1, D2, D3 and D4 are named as CH1, CH2, $\mathrm{CH} 3$ and $\mathrm{CH} 4$, respectively, and the backscattered signals from $\mathrm{L} 2$, recorded at D1, D2, D3 and D4 are named as $\mathrm{CH} 5, \mathrm{CH} 6, \mathrm{CH} 7$ and $\mathrm{CH} 8$, respectively. The ratios used are $\ln (\mathrm{CH} 1 / \mathrm{CH} 2)$, $\ln (\mathrm{CH} 3 / \mathrm{CH} 4), \ln (\mathrm{CH} 5 / \mathrm{CH} 6)$ and $\ln (\mathrm{CH} 7 / \mathrm{CH} 8)$.

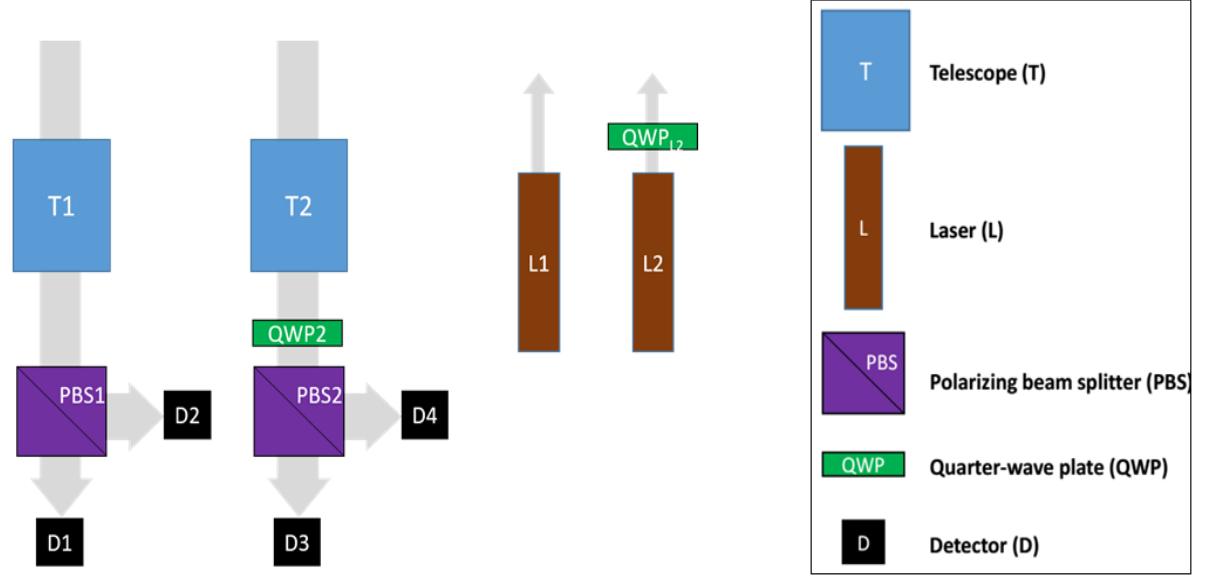

Figure 1: The polarizing lidar design. 


\subsection{Simulated dataset for system evaluation}

The system capabilities and limitations in detecting the percentage of oriented dust particles are investigated using simulated atmospheric scenarios, generated from a system signal simulator and considering the scattering properties of dust particles with realistic irregular shapes and their mixtures as oriented and randomly-oriented particles. The scattering database is generated considering the irregular-shaped particles of [8] (Fig. 2), using the ADDA code and the extensive computational resources of MareNostrum HPC and ARIS HPC.

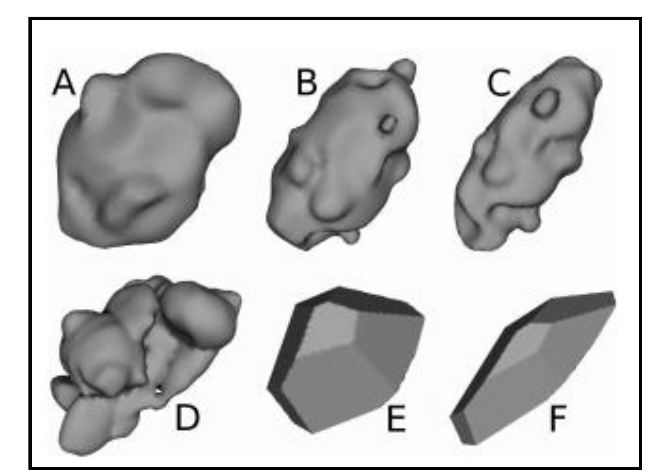

Figure 2: Particle shapes considered for dust particles (Source: [8]).

The maximum dust volume-equivalent size parameter considered is $\sim 60$ and the particle refractive indices are $1.6+\mathrm{i} 0$ for "non absorbing" dust and 1.48+i0.002 for "absorbing" dust. We make the assumption that the particles are not oriented at fixed angles, but "wobble" over a range of angles along the vertical direction. Moreover, we consider various heights above ground and depths for the dust layers, and we omit the presence of other aerosol layers in the atmospheric scene. The AODs of the dust layers range from 0.05 to 1.5 at 532 and $1064 \mathrm{~nm}$. The above assumptions may be over-simplifications of the realistic atmospheric scenes, but they are useful as a first step towards a more extensive analysis.

\section{RESULTS}

\subsection{Information content for the percentage of oriented particles}

Figure 3 presents a preliminary analysis of the information content for the percentage of oriented dust particles in the simulated signals considered. The analysis is done for a system emitting at 532 $\mathrm{nm}$ (Fig. 3a) and at $1064 \mathrm{~nm}$ (Fig. 3b) and performing measurements at $60^{\circ}$ from zenith. It seems that the measurements at $1064 \mathrm{~nm}$ provide more information on the dust particle orientation. These results will be further analyzed considering the real SNR of 1064 and $532 \mathrm{~nm}$ detectors, taking also into account viewing angles at e.g. $30^{\circ}$ and $45^{\circ}$ from zenith. Finally, they will be used for constructing a retrieval scheme for the properties of oriented dust particles. 

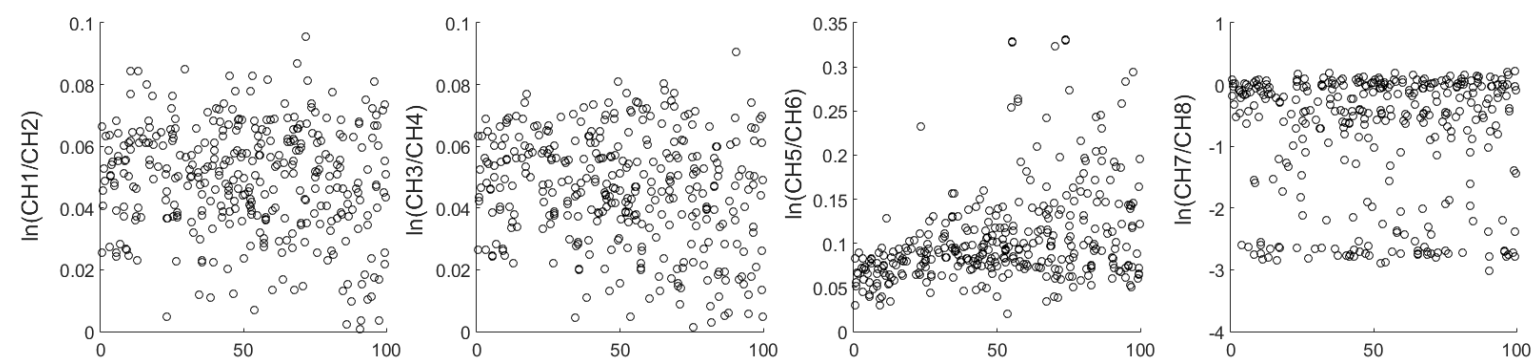

a)
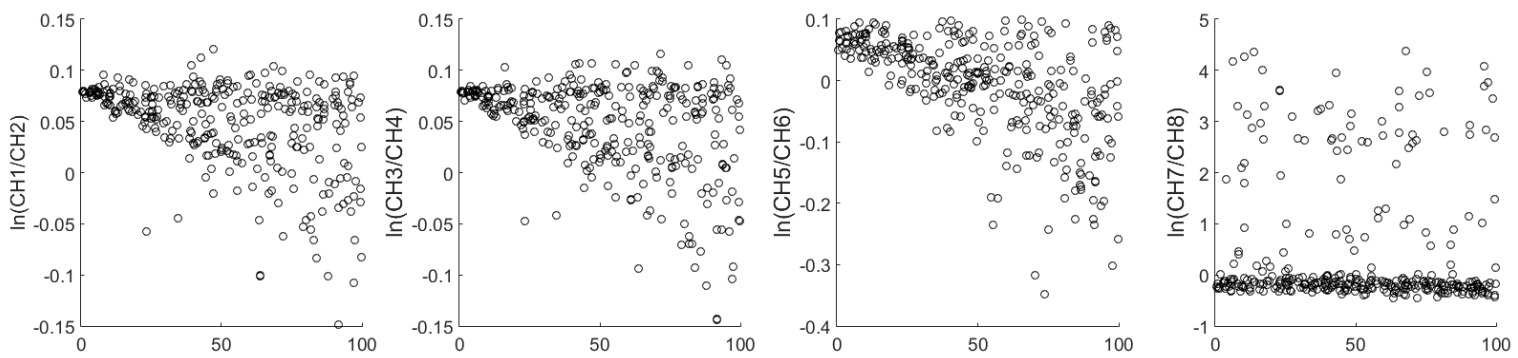

b)

Figure 3: a) Percentage of oriented dust particles in the dust layers of the simulated atmospheric scenes, plotted versus the ratios of the logarithms of the signals (from left to right) $\ln (\mathrm{CH} 1 / \mathrm{CH} 2), \ln (\mathrm{CH} 3 / \mathrm{CH} 4)$, $\ln (\mathrm{CH} 5 / \mathrm{CH} 6)$ and $\ln (\mathrm{CH} 7 / \mathrm{CH} 8)$, for backscattered light at $532 \mathrm{~nm}$, viewed by the lidar with $60^{\circ}$ from zenith. b) The same with (a), for backscattered light at $1064 \mathrm{~nm}$.

\section{ACKNOWLEDGEMENTS}

This work was supported by the European Research Council under the European Community's Horizon 2020 research and innovation framework program/ERC grant agreement 725698 (D-TECT). The authors acknowledge PRACE for awarding access to MareNostrum at Barcelona Supercomputing Center (BSC), Spain, and GRNET for awarding computational time in the National HPC facility ARIS - under project ID pa170906-ADDAPAS and pr005038-REMOD.

\section{REFERENCES}

[1] Z. Ulanowski, et al., Atmos. Chem. Phys. 7: 61616173 (2007)

[2] M. Geier and M. Arienti, J Quant Spectrosc Radiat Transf 149: 16-32 (2014)

[3] Y.Balin et al., Opt. Express, 21(11): 13408-13418 (2013)

[4] V. Bruno et al., Appl. Opt., OSA, 43: 6620-6628 (2004)

[5] S. N. Volkov et al., Appl. Opt., OSA, 54: $3095-$ 3105 (2015)

[6] V. Freudenthaler and T. Berkoff, Proc. of 2nd GALION workshop, WMO, Geneva (2010)

[7] M. A. Yurkin and A. G. Hoekstra, J. Quant. Spectrosc. Radiat., 112: 2234-2247 (2011)

[8] J. Gasteiger et al., Tellus B, 63: 725-741 (2011) 